\title{
A Tuberculosis Outbreak During the COVID-19 Pandemic - Hubei Province, China, 2020
}

\author{
Dan $\mathrm{Li}^{1}$; Xiaowang Peng ${ }^{2}$; Shuangyi $\mathrm{Hou}^{3}$; Tao Li ${ }^{4,4,}$; Xue-jie $\mathrm{Yu}^{1, * *}$
}

\section{Summary \\ What is already known about topic? \\ Multidrug-resistant tuberculosis (MDR-TB) has become a growing threat to public health. There were few reports about family-to-school MDR-TB outbreaks in China, especially during the coronavirus disease 2019 (COVID-19) pandemic. \\ What is added by this report? \\ A tuberculosis (TB) outbreak happened in Hubei Province during the COVID-19 pandemic. The transmission chain was probably from a father (MDR- TB case) with retreated TB history to his daughter, who then spread $\mathrm{TB}$ to her classmates. \\ What are the implications for public health practice? \\ We should enhance TB control both in schools and households, including strengthening TB/MDR-TB detection, health education, and ventilation. The TB contact screening cannot only be limited to outside school settings and should be conducted in the school when a TB student is absent from school for 2 or 3 months, or even longer especially during the COVID- 19 pandemic.}

On April 17, 2020, 10 days after the coronavirus disease 2019 (COVID-19) pandemic lockdown ended and before schools reopened in Hubei Province, China, a hospital reported a student tuberculosis (TB) pleurisy case. The local CDC verified the case and conducted close contact screening and epidemiological investigations immediately. As a result, 6 active TB cases including 5 students $[1$ multidrug-resistant tuberculosis (MDR-TB) case, 1 rifampin-resistant TB (RR-TB) case, and 3 clinically-diagnosed TB case] and 1 student's father (MDR-TB) were identified.

\section{INVESTIGATION AND RESULTS}

The outbreak occurred at a senior high school with a total of 576 students and 82 staff members. The index case was a 17-year-old female student in Grade 2 (equivalent to 11th Grade), who complained of left side chest pain while breathing since March 10, 2020, during the COVID-19 pandemic lockdown, and was clinically diagnosed as TB pleurisy with left pleural effusion through chest X-ray on April 17 at a local TB designated hospital. Epidemiological investigation showed she stayed at home without going out from January 15 to the onset date because of the COVID-19 pandemic and community lockdown and had no history of $\mathrm{TB}$ clinical symptoms in the previous semester.

Close contact screening was first performed in the household of the index case. Household contacts including parents were excluded for TB because of lack of clinical symptoms and had negative laboratory test results. Then, TB screening was performed among classmates and faculty who shared a classroom with the index case according to the National Norms of Tuberculosis Prevention and Control in School (2017) (1). As a result, 20 out of 82 students (24.4\%) and 1 of 9 faculty $(11.11 \%)$ had strong positive purified protein derivatives tuberculosis (PPD) with tuberculin skin test (TST) without difference (Fisher's exact: 1 tailed $P=0.336$ ). Among 20 strong positive PPD students, further GeneXpert and anti-TB drug sensitivity test (DST) indicated 4 were active pulmonary TB including 1 MDR-TB resistant to rifampin (R), isoniazid $(\mathrm{H})$, and ofloxacin (O); 1 RR-TB (GeneXpert MTB/RIF positive with negative culture result); and 2 clinically-diagnosed TB. The rest of the faculty $(\mathrm{n}=1)$ and students $(\mathrm{n}=16)$ with strong positive PPD were diagnosed as latent tuberculosis infection (LTBI). All TB cases in this outbreak were diagnosed and classified according to the national TB diagnosis and classification standards (2-3). The screening was expanded to students and faculty members who have classrooms on the same floor with the TB cases, and 1 out of the 78 students (1.3\%) was diagnosed as LTBI (Figure 1).

The total attack rate among students after close contact screening was $2.5 \%(4 / 160)$ for active $\mathrm{TB}$ 


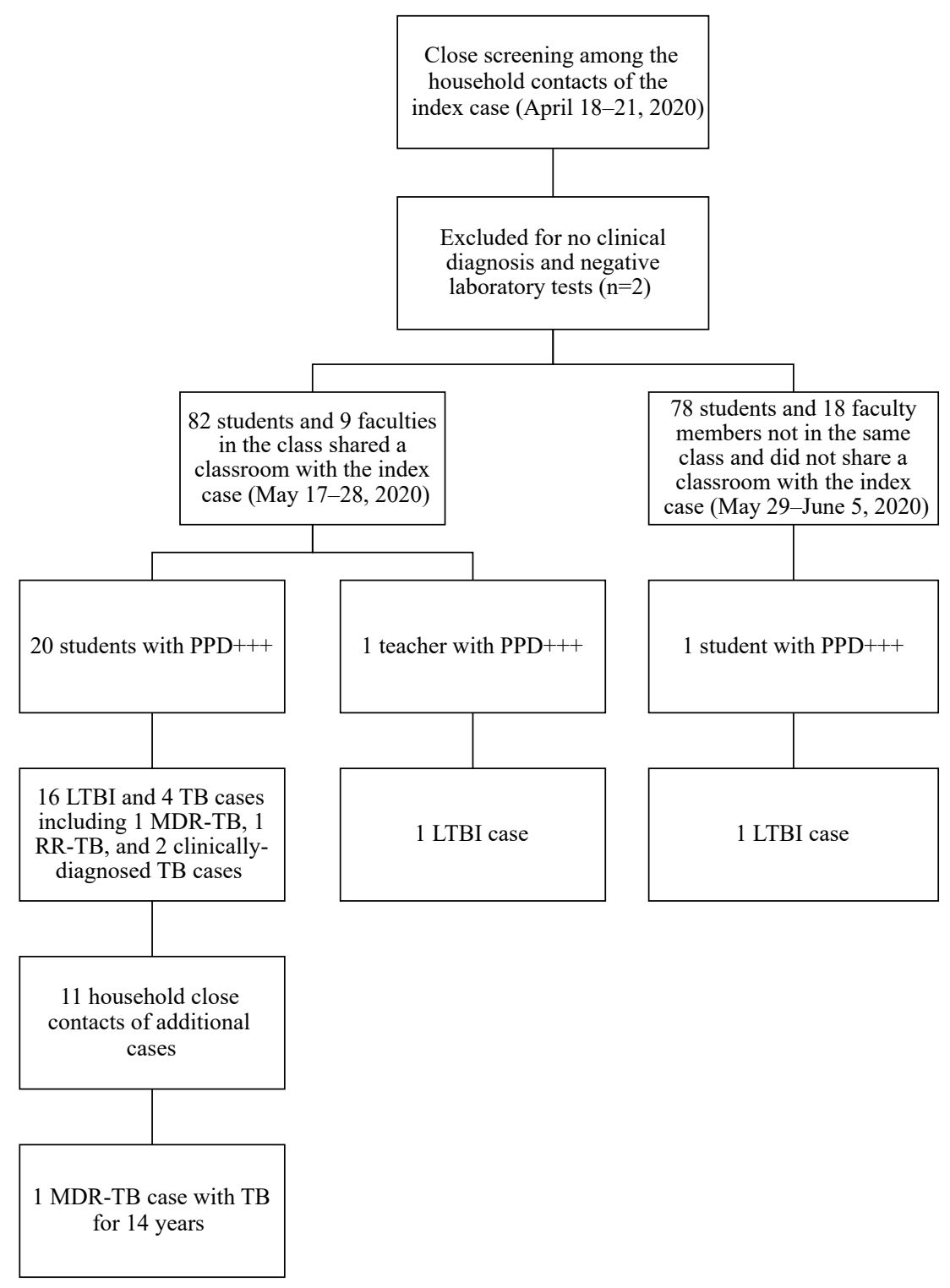

FIGURE 1. The flow chart of close contact screening and case finding of the MDR-TB outbreak - Hubei Province, China, 2020.

Note: PPD+++, strong positive PPD with induration above or equal to $15 \mathrm{~mm}$ and/or blisters, lymphangitis, or necrosis.

Abbreviations: MDR-TB=multidrug-resistant tuberculosis; $\mathrm{TB}=$ tuberculosis; $\mathrm{RR}-\mathrm{TB}=$ rifampin resistant tuberculosis; $\mathrm{LTBI}=$ latent tuberculosis infection; $n=$ number.

cases, 13.1\% (21/160) for strong positive PPD, and $10.6 \%$ (17/160) for LTBI cases. For students sharing the same classroom with the index-case, the attack rate was $4.9 \%$ (4/82) for active TB cases, $24.4 \%$ (20/82) for strong positive PPD, and $19.5 \%$ (16/82) for LTBI cases. The risk ratio (RR) of being strong positive PPD and LTBI in the shared classroom with the index case was 19 and 15 times higher, respectively, than that of students who did not share the same classroom as the index patient. No active TB cases were identified in students who did not share the same classroom with index-case on the same floor.
Epidemiological investigation showed these 4 additional active TB cases, identified among students who shared the same classroom with the index case, stayed at home and scarcely went out from January 15 to May 17 (TB screening began) because of the COVID-19 pandemic lockdown. Among these 4 additional active $\mathrm{TB}$ cases, the MDR-TB student patient experienced a chronic cough for a half year since late November 2019 and had attended school for 2 months since she became ill until the school holiday on January 15, 2020. She did not declare illness in the school or visite a doctor, but only self-medicated by 
using common cough medicine. Retrospective investigation in her family showed that her father had a TB history for 14 years with 3 recorded times of hospital TB treatment records before the current investigation (2007, 2011, and 2013). He was eventually diagnosed as an MDR-TB case with the same drug resistance $(\mathrm{R}, \mathrm{H}$, and $\mathrm{O}$ resistant) in later May 2020. When investigating the TB-related knowledge in this family, members showed poor awareness about $\mathrm{TB}$ transmission and the potential threats.

The MDR-TB student had clinical symptoms earlier than the index case and was likely the most probable initial case in her class. She had a cough for 2 months since November 2019 while attending school. From November 2019 to January 2020, it was winter and the local temperature ranged from 1 to $10{ }^{\circ} \mathrm{C}$. During the wintertime, the school used air conditioning units to heat classrooms and rarely opened doors and windows for ventilation, which might have promoted the spread of mycobacterium tuberculosis (MTB) in the classroom. The school also failed on routine prevention and control measures such as morning and noon inspection of students for illnesses. The teachers and students did not have strong knowledge on suspected TB symptoms, which delayed detection and treatment.

All active TB cases were treated and managed by municipal and provincial expert groups, and suspension and resumption of school attendance were implemented according to national guidelines (1). All contacts with strong positive PPD reactions and normal chest X-ray results were routinely monitored and given chest X-ray reexaminations at 3, 6, and 12 month follow-ups. The results of follow-up checks showed that students and staff with strong positive of PPD had not developed any active TB as of June 15, 2021.

\section{DISCUSSION}

We reported a TB outbreak in a senior high school in Hubei Province, China, 2020. The cases were diagnosed 10 days later after the COVID-19 pandemic lockdown and before schools reopened. Although lacking of gene homology verification, the outbreak was identified based on field investigation and drug resistance results as a family-to-school related outbreak of MDR-TB. All likely subsequent TB cases were in the same class. Among the 17 LTBI cases, 16 cases were found in students sharing the same classroom with the index patient and there was only 1 exception. The risk ratio of being strongly positive PPD and LTBI in the students who were in the same classroom with the index patient was significantly higher than that of students who were not in the same classroom with the index patient. All 3 confirmed TB cases in this study were drug-resistant (2 MDR-TB and 1 RRTB). More than $75 \%$ ( $82 \%$ in $2017,78 \%$ in 2018 , and $78 \%$ in 2019) of RR-TB had MDR-TB according to Global Tuberculosis Report. Considering the illness history and close contact history of each case, it was assumed that the MDR-TB student got TB from her father and disseminated TB to her classmates, although other sources of infection could not be excluded due to lack of genetic testing.

Our research has exposed some problems and challenges of $\mathrm{TB}$ prevention and control. First, the MDR-TB student's father had TB for 14 years, but he was never diagnosed as MDR-TB until this investigation. The father had records of three previous hospital TB treatment courses before this investigation but was likely not identified because drug sensitivity tests are not routinely carried out in this area. Second, the MDR-TB student's father had a long chronic TB history, but his low awareness of TB-related knowledge suggested a lack of public health education in local society. Third, the first MDR-TB student had a cough for 2 months, but the student continued to attend school until the winter holiday. This put other students at risk of TB/MDR-TB, indicating a lack of knowledge of $\mathrm{TB}$ prevention among senior middle students and faculty members. Finally, the ventilation of the classroom was insufficient. Two air conditioning units that could provide heating were used in the winter in the classroom with inadequate frequency of opening the window for ventilation. As a result of high-speed economic development, most schools are equipped with air conditioners in China, and the lower ventilation may increase risk of transmission of TB.

There were few reports about the outbreak of MDR$\mathrm{TB}$ in China, especially during the COVID-19 pandemic (4-5). Due to the COVID-19 lockdown, the index TB case was absent from school for about three months prior to diagnosis. This outbreak indicated that $\mathrm{TB}$ contact screening cannot be ignored in the school when a student was diagnosed as a TB case although she/he may be absent from school for 2 or 3 months. Although genotyping methods to investigate the source in a school TB outbreak were effective, lack of gene homology verification due to the COVID-19 pandemic and restrictions on local 
laboratory conditions was the limitations in this study.

This family and school related outbreak of MDRTB was caused by an absence of MDR-TB diagnosis knowledge and lack of overall TB knowledge. We suggest sustained efforts should be made to enhance TB health education in the community on topics such as transmission and common symptoms. We also suggest strengthening $\mathrm{TB}$ control mechanism in schools including morning inspections, illness tracing, and infection control activities (e.g., ventilation especially for air-conditioned places).

Acknowledgments: Local health sector and education sector.

Funding: The National Natural Science Funds of China (Grant Numbers: 81971939).

doi: $10.46234 / \mathrm{ccdcw} 2021.145$

\# Corresponding authors: Tao Li, litao1@chinacdc.cn; Xue-jie Yu, yuxuejie@whu.edu.cn.

\footnotetext{
${ }^{1}$ State Key Laboratory of Virology, School of Health Sciences, Wuhan University, Wuhan, Hubei, China; ${ }^{2}$ Institute of tuberculosis control, Kashi Center for Disease Control and Prevention, Kashi, Xinjiang Uygur Autonomous Region, China; ${ }^{3}$ Hubei Provincial Center for Disease Control and Prevention, Wuhan, Hubei, China; ${ }^{4}$ National
}

Center for Tuberculosis Control and Prevention, Chinese Center for Disease Control and Prevention, Beijing, China.

Submitted: June 03, 2021; Accepted: June 24, 2021

\section{REFERENCES}

1. General Office of National Health and Family Planning Commission, General Office of the Ministry of Education. National norm of tuberculosis prevention and control in school (2017). 2017. http://www. moe.gov.cn/srcsite/A17/moe_943/s3285/201707/t20170727_310182.ht ml. [2021-5-8]. (In Chinese).

2. National Health Commission of the People's Republic of China. WS 288-2017 national diagnosis for pulmonary tuberculosis. 2017 http://www.nhc.gov.cn/wjw/s9491/201712/a452586fd21d4018b0ebc00 b89c06254.shtml. [2021-5-8]. (In Chinese).

3. National Health Commission of the People's Republic of China. WS 196-2017 classifica-tion of tuberculosis. 2017. http://www.nhc.gov.cn/ wjw/s9491/201712/0d3c52de984b4bc4add047f19ccd51b9.shtml. [2021-5-8]. (In Chinese).

4. Wang SL, Cui Y, Liu NQ, Wang XJ, Wang XQ, Wu WD, et al. A tuberculosis outbreak at a school - Xinjiang Uygur autonomous region, China, 2019. China CDC Wkly 2020;2(46):881 - 3. http://dx.doi.org/ $10.46234 / \mathrm{ccdcw} 2020.240$.

5. Zhang MX, Wang T, Hou SY, Ye JJ, Zhou LP, Zhang Z, et al. An outbreak of multi-drug-resistant tuberculosis in a secondary school Hubei province, 2019. China CDC Wkly 2019;1(5):67 - 9. http://dx. doi.org/10.46234/ccdcw2019.020. 